\title{
The automation of processes of atlas mapping
}

\author{
Krylov S.A. ${ }^{\text {a }}$ *, Zagrebin G.I. ${ }^{\text {b, }}$, Dvornikov A.V. ${ }^{\mathrm{c}}$, Loginov D.S. $^{\mathrm{d}}$, Fokin I.E. ${ }^{\mathrm{e}}$ \\ ${ }^{a}$ Moscow State University of Geodesy and Cartography (MIIGAiK), krylov@cartlab.ru, \\ ${ }^{b}$ Moscow State University of Geodesy and Cartography (MIIGAiK), gleb@cartlab.ru, \\ ${ }^{c}$ Moscow State University of Geodesy and Cartography (MIIGAiK), antondvk@cartlab.ru, \\ ${ }^{d}$ Moscow State University of Geodesy and Cartography (MIIGAiK), loginov@cartlab.ru, \\ ${ }^{e}$ Moscow State University of Geodesy and Cartography (MIIGAiK), fokin@ cartlab.ru \\ * Corresponding author
}

Keywords: atlas, atlas mapping, atlas structure, automatization, cartographic database, geographic base maps for atlas, mathematical basis, reference information, spatial-temporary data

\begin{abstract}
:
Atlas mapping is one of the priorities of the modern mapping industry. It is connected both with the unceasing popularity of traditional atlases in the printing version, and with the active introduction and use of interactive electronic and web atlases, as well as with the development of atlas information systems. Currently there are no generalizing methodological solutions for automating the atlas compilation processes, despite the importance and perspectives of atlas mapping. In addition, there is no software which ensures all requirements for the design of the atlas. The use of geographic information technologies in atlas mapping doesn't solve all the problems involved in creating atlases. For example, GIS can be used for solution of individual processes, e.g. the development of spatial and thematic databases, the creation of thematic maps, the cartographic generalization, the project of graphical index of maps into atlas pages, etc. But GIS doesn't provide for the creation of a finished cartographic work with an integrated structure and content. This fact undoubtedly leads to the high cost and laboriousness of creating atlases, to the long-term design and the presence of errors in their compilation.
\end{abstract}

The Department of Cartography of the Moscow State University of Geodesy and Cartography (MIIGAiK) carries out research work on the development of the theory and methods of automating atlas mapping processes. The research is being directed to the speed of creation and improvement of the quality of general geographical, thematic and complex atlases. As part of this research, methods and algorithms for the automated creation of atlases have been developed, providing solutions to the most time-consuming and rather complex processes of atlas mapping, among which are:

1) The development of an optimal atlas structure;

2) Design of the mathematical basis of the atlas (selection of a scale series, cartographic projections, format and layout);

3) Formalization of the creation of geographic base maps for atlas;

4) The formation and visualization of reference information of the atlas;

5) Organization, storage and use of spatial-temporary data in electronic atlases.

Figure 1 presents the proposed solutions for automating processes mentioned above. The list of solutions may be different depending on the type of atlas being created (general geographical, thematic, and complex atlas). So the following options can be when developing the structure of the atlas. The use of a unified system of classification and coding of thematic maps and taking into account the degree of knowledge (study) of the object and mapped phenomenon is being used only for a thematic or complex regional atlas. The definition of possible combinations of territories shown on a single atlas map is used only for thematic or general geographical atlas. For each type of atlas, the formation and use of the reference information system of the studied atlases can be used, as well as the identification and formalization of factors affecting the inclusion of a specific section or a separate map.

The results of the research will ensure the efficiency of creating atlases and increase their quality. Also its will help to meet the increasing consumer demand for atlas map products, especially in the form of electronic atlases and geo-portal solutions. 
The development of an optimal atlas structure

\begin{tabular}{|c|}
\hline The use of a unified system of classification and coding of thematic maps \\
\hline Formation and use of the reference and search system of studied atlases \\
\hline Definition and formalization of factors affecting the inclusion of particular section or separate map to the atlas \\
\hline Accounting of study degree of mapped object and phenomenon \\
\hline Definition of possible combinations of territories (states), displayed on a single map \\
\hline
\end{tabular}

\section{Designing a mathematical basis for atlas}

Formalization of the relationship of the map position in the atlas structure and its map projection

Determination of the scale series of maps $\quad$ Selection of cartographic projections

Definition of the frequency of cartographic grids and their construction

Orientation and layout determination

Formalization of the creation of geographical base maps of the atlas

Definition of the number and list of created typical geographic base maps

Determination of quantitative indicators of the base maps (density and graphical load)

depending on the legend size and cartographic method

Coordination of geographical objects with thematic content of maps

Formation of the selection table of objects for each geographic base maps

Formation and visualization of atlas reference information

Automation of the compilation and design processes for structural diagrams, graphs and diagrams

Rising of informativeness of reference documents

Consideration of the significance of objects, their spatial relationships and cartometric characteristics

Construction of diagrams and charts, cartographic illustrations

taking into account the geographical features of the mapped territory with optimal scale and projection

Organization, storage and use of spatial-temporal data in electronic atlases

Tracking information changes over time

Data storage in PostgreSQL with PostGIS spatial extension

Storage of multimedia data in file databases with time stamp and geolocation

Use automatic search for information on the Internet and save data in

specialized database with indexing information on time and space

Spatial Indexing

Figure 1. The proposed solutions for automating processes of atlases creating.

\section{Acknowledgments:}

This work was supported by Ministry of education and science of Russian Federation, project 5.8029.2017/8.9 